\title{
A LINK BETWEEN HARMONICITY OF 2-DISTANCE FUNCTIONS AND INCOMPRESSIBILITY OF CANONICAL VECTOR FIELDS
}

\author{
BANG-YEN CHEN
}

\begin{abstract}
Let $M$ be a Riemannian submanifold of a Riemannian manifold $\tilde{M}$ equipped with a concurrent vector field $\tilde{Z}$. Let $Z$ denote the restriction of $\tilde{Z}$ along $M$ and let $Z^{T}$ be the tangential component of $Z$ on $M$, called the canonical vector field of $M$. The 2distance function $\delta_{Z}^{2}$ of $M$ (associated with $Z$ ) is defined by $\delta_{Z}^{2}=\langle Z, Z\rangle$.

In this article, we initiate the study of submanifolds $M$ of $\tilde{M}$ with incompressible canonical vector field $Z^{T}$ arisen from a concurrent vector field $\tilde{Z}$ on the ambient space $\tilde{M}$. First, we derive some necessary and sufficient conditions for such canonical vector fields to be incompressible. In particular, we prove that the 2-distance function $\delta_{Z}^{2}$ is harmonic if and only if the canonical vector field $Z^{T}$ on $M$ is an incompressible vector field Then we provide some applications of our main results.
\end{abstract}

\section{Incompressible vector fields}

In fluid mechanics, many liquids are hard to compress (i.e., their volumes or densities don't change much when you squeeze them), so that the density $\rho$ is essentially a constant. For such an incompressible fluid the equation of continuity simplifies to the divergence of the flow velocity $v$ is zero, i.e.,

$$
\operatorname{div}(v)=0 \quad \text { (incompressible) }
$$

so that the velocity field ${ }^{1} v$ is an incompressible vector field (also known as a solenoidal vector field or a divergence-free vector field). This condition is analogous to the condition $\operatorname{div}(B)=0$ in electromagnetism that the magnetic field $B$ has zero divergence.

It is well-known that incompressible vector fields are important in magnetohydrodynamics. Moreover, magnetic fields are widely used throughout modern technology, particularly in electrical engineering and electromechanics (cf. e.g., [1, 15, 16]).

Based on the reasons mentioned above, one has the following.

Received Arpil 2, 2018, accepted June 8, 2018.

2010 Mathematics Subject Classification. Primary 31A05; Secondary 53C42, 76D99.

Key words and phrases. Concurrent vector field, canonical vector field, incompressible vector field, 2-distance function; harmonic function.

${ }^{1}$ All vector fields, functions, immersions and manifolds are assumed to be smooth. 
Definition 1.1. A vector field $X$ on a Riemannian manifold $M$ is called incompressible if the divergence of $X$ is zero, i.e., $\operatorname{div}(X)=0$.

Let $\phi: M \rightarrow \tilde{M}$ be an isometric immersion of a Riemannian manifold $M$ into another Riemannian manifold $\tilde{M}$. Denote by $\langle$,$\rangle the inner product of M$ as well as of $\tilde{M}$. Assume that $\tilde{Y}$ is a vector field of $\tilde{M}$. Denote by $Y$ the restriction of $\tilde{Y}$ along $M$. Then $Y$ admits an orthogonal decomposition:

$$
Y=Y^{T}+Y^{\perp}
$$

where $Y^{T}$ and $Y^{\perp}$ are the tangential and the normal components of $Y$, respectively. The tangent vector field $Y^{T}$ of $M$ is called the canonical vector field of $M$ associated with $Y$.

For a submanifold $M$ of a Euclidean space $\mathbb{E}^{m}$, the most elementary and natural vector field on $M$ is the position vector field $\mathbf{x}$. The tangential component $\mathbf{x}^{T}$ of $\mathbf{x}$ is simply called the canonical vector field of $M[11,12]$. It is well-known that the position vector field of $\mathbb{E}^{m}$ is a concurrent vector field (see Definition 2.2 and Example 2.1).

In earlier articles, we have investigated Euclidean submanifolds whose canonical vector fields are concurrent [6, 8], concircular [14], torse-forming [13], conformal [12], or incompressible [11]. (See also recent surveys $[9,10]$ for several topics on position vector fields on Euclidean submanifolds.)

In this article, we initiate the investigation of submanifolds $M$ of $\tilde{M}$ with incompressible canonical vector field $Z^{T}$ arisen from a concurrent vector field $\tilde{Z}$ on the ambient space $\tilde{M}$. First, we derive some necessary and sufficient conditions for such canonical vector fields to be incompressible. In particular, we prove that the 2-distance function $\delta_{Z}^{2}$ is harmonic if and only if the canonical vector field $Z^{T}$ on $M$ is an incompressible vector field. Then we provide some applications of our main results.

\section{Preliminaries}

Let $\phi: M \rightarrow \tilde{M}$ be an isometric immersion of a connected Riemannian $n$-manifold $M$ into a Riemannian $m$-manifold $\tilde{M}$. For each point $p \in M$, we denote by $T_{p} M$ and $T_{p}^{\perp} M$ the tangent space and the normal space of $M$ at $p$, respectively. Let $\nabla$ and $\tilde{\nabla}$ denote the LeviCivita connections of $M$ and $\mathbb{E}^{m}$, respectively.

The formula of Gauss and the formula of Weingarten are then given respectively by (cf. [3, $4,7])$

$$
\begin{aligned}
& \tilde{\nabla}_{X} Y=\nabla_{X} Y+h(X, Y), \\
& \tilde{\nabla}_{X} \xi=-A_{\xi} X+D_{X} \xi,
\end{aligned}
$$


for vector fields $X, Y$ tangent to $M$ and $\xi$ normal to $M$, where $h$ denotes the second fundamental form, $D$ is the normal connection and $A$ is the shape operator of $M$.

For each normal vector $\xi$ at $p$, the shape operator $A_{\xi}$ is a self-adjoint endomorphism of $T_{p} M$. The second fundamental form $h$ and the shape operator $A$ are related by

$$
\left\langle A_{\xi} X, Y\right\rangle=\langle h(X, Y), \xi\rangle .
$$

The mean curvature vector field $H$ of an $n$-dimensional submanifold $M$ is defined by

$$
H=\left(\frac{1}{n}\right) \text { trace } h \text {. }
$$

Let $\left\{e_{1}, \ldots, e_{n}\right\}$ be an orthonormal frame on $M$, then the divergence of a vector field $X$ on $M$, denoted by $\operatorname{div}(X)$, is defined by

$$
\operatorname{div}(X)=\sum_{j=1}^{n}\left\langle\nabla_{e_{i}} X, e_{i}\right\rangle .
$$

The gradient $\nabla f$ of a function $f$ on $M$ is defined by

$$
\langle\nabla f, Y\rangle=Y f
$$

for any vector $Y$ tangent to $M$. Hence, in terms of an orthonormal frame $\left\{e_{1}, \ldots, e_{n}\right\}$ on $M$, we have

$$
\nabla f=\sum_{i=1}^{n}\left(e_{i} f\right) e_{i} .
$$

And the Laplacian $\Delta$ of $M$ acting on a function $f$ on $M$ is given by

$$
\Delta f=-\sum_{i=1}^{n}\left\{e_{i} e_{i}(f)-\nabla_{e_{i}} e_{i}(f)\right\} .
$$

Now, we present some basic definitions for later use.

Definition 2.2. A vector field $\tilde{Z}$ on a Riemannian manifold $\tilde{M}$ is called a concurrent vector field if it satisfies

$$
\tilde{\nabla}_{X} \tilde{Z}=X
$$

for all vectors $X$ tangent to $\tilde{M}$, where $\tilde{\nabla}$ denotes the Levi-Civita connection of $\tilde{M}$ (cf. [19, 20])

Concurrent vector fields play some important roles in differential geometry and mathematical physics. For instance, it was proved in [19] that if the holonomy group of a Riemannian manifold $\tilde{M}$ leaves a point invariant, then $\tilde{M}$ admits a concurrent vector field. Concurrent vector fields have also been studied in Finsler geometry since the beginning of 1950s (cf. $[17,18])$.

The simplest example of Riemannian manifold with a concurrent vector field is a Euclidean space. 
Example 2.1. The position vector field $\mathbf{x}$ of the Euclidean $m$-space $\mathbb{E}^{m}$ is a concurrent vector field.

Definition 2.3. Let $B$ and $F$ be two Riemannian manifolds of positive dimensions equipped with metrics $g_{B}$ and $g_{F}$, respectively, and let $f$ be a positive smooth function on $B$.

The warped product $M=B \times{ }_{f} F$ is the product manifold $B \times F$ equipped with the warped product metric

$$
g=g_{B}+f^{2} g_{F}
$$

The function $f$ is called the warping function of the warped product (cf. $[2,11])$.

For a warped product $B \times{ }_{f} F, B$ is called the base and $F$ the fiber. The leaves $B \times\{q\}=$ $\eta^{-1}(q), q \in F$, and the fibers $\{b\} \times F=\pi^{-1}(p), b \in B$ are Riemannian submanifolds of $B \times{ }_{f} F$.

Example 2.2. It is direct to verify that $\mathbb{E}_{*}^{m}=\mathbb{E}^{m}-\{0\} \subset \mathbb{E}^{m}$ can be regarded as the warped product $\mathbf{R}^{+} \times{ }_{s} S^{m-1}$ equipped with the warped product metric

$$
g=d s^{2}+s^{2} g_{S}
$$

where $g_{S}$ is the metric tensor of the unit $(m-1)$-sphere $S^{m-1}$. In this case, the position vector field $\mathbf{x}$ of $\mathbb{E}_{*}^{m}$ is given by $s \frac{\partial}{\partial s}$.

The distance function $\delta$ from the origin $o \in \mathbb{E}^{m}$ to a point of $\mathbb{E}^{m}$ is given by

$$
\delta=\sqrt{\langle\mathbf{x}, \mathbf{x}\rangle}
$$

Example 2.3. Let $F$ be any Riemannian manifold and let $I=(a, b)$ be an open interval with $0 \notin I$. Consider the warped product $I \times{ }_{s} F$ equipped with the warped product metric

$$
\tilde{g}=d s^{2}+s^{2} g_{F}
$$

where $g_{F}$ denotes the Riemannian metric of $F$. Then the vector field $\tilde{Z}=s \frac{\partial}{\partial s}$ is a concurrent vector field on $I \times{ }_{s} F$ (cf. Example 3.1 of [5]). Moreover, in this case the vector field $\tilde{Z}=s \frac{\partial}{\partial s}$ can be considered as the radial vector field of $I \times{ }_{s} F$.

\section{Theorems}

Now, we define the notion of $r$-distance function on a submanifold $M$ of a Riemannian manifold $\tilde{M}$ equipped with a concurrent vector field as follows. 
Definition 3.4. Let $M$ be a submanifold of a Riemannian manifold $\tilde{M}$ equipped with a concurrent vector field $\tilde{Z}$. Denote by $Z$ the restriction of $\tilde{Z}$ on $M$. Then the function

$$
\delta_{Z}^{r}(p)=\left|Z_{p}\right|^{r}=\left\langle Z_{p}, Z_{p}\right\rangle^{r / 2}
$$

is called the $r$-distance function (associated with $Z$ ) (or simply the $r$-distance function if there is no confusion arisen).

Lemma 3.1. Let $M$ be a submanifold of a Riemannian manifold $\tilde{M}$ equipped with a concurrent vector field $\tilde{Z}$ on $\tilde{M}$. Then the corresponding canonical vector field $Z^{T}$ and the gradient of the 2-distance function $\delta_{Z}^{2}$ of $M$ are related by

$$
Z^{T}=\frac{1}{2} \nabla \delta_{Z}^{2}
$$

Proof. Let $M$ be a submanifold of a Riemannian manifold $\tilde{M}$ equipped with a concurrent vector field $\tilde{Z}$ on $\tilde{M}$. Then the 2-distance function $\delta_{Z}^{2}$ of $M$ is given by

$$
\delta_{Z}^{2}=\langle Z, Z\rangle
$$

where $Z$ is the restriction of $\tilde{Z}$ on $M$.

Let $\left\{e_{1}, \ldots, e_{n}\right\}$ be an orthonormal local frame on $M$. Then it follows from (2.6), (2.8) and (3.2) that

$$
\begin{aligned}
\nabla \delta_{Z}^{2} & =\sum_{i=1}^{n}\left(e_{i}\langle Z, Z\rangle\right) e_{i}=2 \sum_{i=1}^{n}\left\langle\tilde{\nabla}_{e_{i}} Z, Z\right\rangle e_{i} \\
& =2 n \sum_{i=1}^{n}\left\langle e_{i}, Z\right\rangle e_{i}=2 Z^{T},
\end{aligned}
$$

which proves (3.1).

The next result provides a simple characterization of an incompressible canonical vector field on a submanifold arisen from a concurrent vector field on its ambient space.

Theorem 3.1. Let $M$ be a submanifold of a Riemannian manifold $\tilde{M}$ with a concurrent vector field $\tilde{Z}$ on $\tilde{M}$. Then the canonical vector field $Z^{T}$ on $M$ is incompressible if and only if the mean curvature vector field $H$ of $M$ in $\tilde{M}$ satisfies

$$
\langle H, Z\rangle=-1
$$

identically.

Proof. Let $M$ be a submanifold of a Riemannian manifold $\tilde{M}$ equipped with a concurrent vector field $\tilde{Z}$ on $\tilde{M}$. Then, according to Definition 3.4, the canonical vector field $Z^{T}$ is the tangential component of the restriction $Z$ of the concurrent vector field $\tilde{Z}$ along $M$. 
Now, let us compute the $\operatorname{divergence} \operatorname{div}\left(Z^{T}\right)$. It follows from (2.1), (2.4), (2.5) and Lemma 3.1 that

$$
\begin{aligned}
\operatorname{div}\left(Z^{T}\right) & =\frac{1}{2} \sum_{i=1}^{n}\left\langle\nabla_{e_{i}} \nabla \delta_{Z}^{2}, e_{i}\right\rangle=\sum_{i, j=1}^{n}\left\langle\nabla_{e_{i}}\left(\left\langle e_{j}, Z\right\rangle e_{j}\right), e_{i}\right\rangle \\
& =\sum_{i, j=1}^{n}\left(\left\langle\tilde{\nabla}_{e_{i}} e_{j}, Z\right\rangle\left\langle e_{j}, e_{i}\right\rangle+\left\langle e_{j}, e_{i}\right\rangle^{2}+\left\langle e_{j}, Z\right\rangle\left\langle\nabla_{e_{i}} e_{j}, e_{i}\right\rangle\right) \\
& =n(1+\langle H, Z\rangle)+\sum_{i, j=1}^{n}\left(\left\langle\nabla_{e_{i}} e_{j}, Z\right\rangle\left\langle e_{j}, e_{i}\right\rangle+\left\langle e_{j}, Z\right\rangle\left\langle\nabla_{e_{i}} e_{j}, e_{i}\right\rangle\right) .
\end{aligned}
$$

Let us put

$$
\nabla_{X} e_{i}=\sum_{k=1}^{n} \omega_{i}^{k}(X) e_{k}
$$

for tangent vectors $X$ of $M$. Then we find from the fact that $\nabla$ is a metric connection that

$$
\omega_{i}^{k}=-\omega_{k}^{i}
$$

for $1 \leq i, k \leq n$.

From (3.5) and (3.6) we obtain

$$
\begin{aligned}
& \sum_{i, j=1}^{n}\left(\left\langle\nabla_{e_{i}} e_{j}, Z\right\rangle\left\langle e_{j}, e_{i}\right\rangle+\left\langle e_{j}, Z\right\rangle\left\langle\nabla_{e_{i}} e_{j}, e_{i}\right\rangle\right) \\
& =\sum_{i, k=1}^{n} \omega_{i}^{k}\left(e_{i}\right)\left\langle e_{k}, \mathbf{x}\right\rangle+\sum_{i, j=1}^{n} \omega_{j}^{i}\left(e_{i}\right)\left\langle e_{j}, Z\right\rangle \\
& =0 .
\end{aligned}
$$

Therefore, after combining (3.4) and (3.7) we have

$$
\operatorname{div}\left(Z^{T}\right)=n\{1+\langle H, Z\rangle\}
$$

Consequently, the canonical vector field $Z^{T}$ is incompressible if and only if $\langle H, Z\rangle=-1$ holds identically.

Remark 3.1. Lemma 3.1 and Theorem (3.1) generalize statement (a) and statement (b) Theorem 3.1 of [11], respectively.

The next result is the main theorem of this article. This main theorem provides a very simple link between harmonicity of the 2-distance function $\delta_{Z}^{2}$ and the incompressibility of the canonical vector field $Z^{T}$. 
Theorem 3.2. Let $M$ be a submanifold of a Riemannian manifold $\tilde{M}$ with a concurrent vector field $\tilde{Z}$. Then the 2-distance function $\delta_{Z}^{2}$ is harmonic if and only if the canonical vector field $Z^{T}$ is incompressible.

Proof. Let $M$ be a submanifold of a Riemannian manifold $\tilde{M}$. Assume that $\tilde{M}$ admits a concurrent vector field $\tilde{Z}$. Let us compute the Laplacian of the 2-distance function $\delta_{Z}^{2}$ of $M$ as follows.

$$
\begin{aligned}
\Delta \delta_{Z}^{2} & =-\sum_{i=1}^{n} e_{i} e_{i}\left(\delta_{Z}^{2}\right)+\sum_{i=1}^{n} \nabla_{e_{i}} e_{i}\left(\delta_{Z}^{2}\right) \\
& =-2 \sum_{i=1}^{n} e_{i}\left\langle e_{i}, Z\right\rangle+2 \sum_{i=1}^{n}\left\langle\nabla_{e_{i}} e_{i} Z, Z\right\rangle \\
& =-2 \sum_{i=1}^{n}\left\langle\tilde{\nabla}_{e_{i}} e_{i}, Z\right\rangle-2 n+2 \sum_{i=1}^{n}\left\langle\nabla_{e_{i}} e_{i} Z, Z\right\rangle \\
& =-2 \sum_{i=1}^{n}\left\langle h\left(e_{i}, e_{i}\right), Z\right\rangle-2 n \\
& =-2 n\{\langle H, Z\rangle+1\} .
\end{aligned}
$$

Now, by combining (3.8) and Theorem 3.1 we obtain the theorem.

For a Euclidean submanifold $M$, if we denote the tangential component of the position vector field $\mathbf{x}$ of $M$ by $\mathbf{x}^{T}$, then $\mathbf{x}^{T}$ is the canonical vector field of the Euclidean submanifold $M$.

For Euclidean submanifolds, Theorem 3.2 yields the following.

Theorem 3.3. Let $M$ be an arbitrary Euclidean submanifold $M$ of $\mathbb{E}^{m}$. Then the canonical vector field $\mathbf{x}^{T}$ of $M$ is incompressible if and only if the 2-distance function $\delta^{2}=\langle\mathbf{x}, \mathbf{x}\rangle$ of $M$ is a harmonic function.

Proof. This is an immediate consequence of Theorem 3.2 since the position vector field $\mathbf{x}$ is a concurrent vector field on $\mathbb{E}^{m}$.

\section{Some applications}

In this section we make the following.

Assumption. Let $M$ be a submanifold of the warped product $\tilde{M}=I \times{ }_{s} F$. We consider the canonical concurrent vector field $\tilde{Z}=s \frac{\partial}{\partial s}$ on $I \times{ }_{s} F$.

Now, we provide the following applications of Theorems 3.1-3.3. 
Corollary 4.1. Let $\tilde{M}=I \times_{s} F$ be a warped product with warped product metric $\tilde{g}=d s^{2}+s^{2} g_{F}$. Then, for every submanifold $B$ of $F$, the canonical vector field $Z^{T}$ of $I \times{ }_{s} B$ is never incompressible.

Proof. Under the hypothesis, the restriction $Z$ of $\tilde{Z}$ on $I \times{ }_{s} B$ is always tangent to $I \times{ }_{s} B$, i.e., $Z^{\perp}=0$. Therefore, condition (2.1) never holds at each point. Consequently, the canonical vector field $Z^{T}$ of $I \times{ }_{s} B$ is never incompressible according to Theorem 3.1.

Corollary 4.2. Let $\tilde{M}=I \times{ }_{s} F$ be a warped product with warped product metric $\tilde{g}=d s^{2}+s^{2} g_{F}$. Then the canonical vector field $Z^{T}$ of every fiber $\left\{s_{o}\right\} \times{ }_{s} F$ in $I \times{ }_{s} F$ is always incompressible.

Proof. Let $M$ be a submanifold of the warped product $\tilde{M}=I \times{ }_{s} F$ endowed with a concurrent vector field $\tilde{Z}=s \frac{\partial}{\partial s}$. Then the 2-distance function of $M$ is given by $\delta_{Z}^{2}=s^{2}$.

Suppose that $M$ is a fiber of $I \times{ }_{s} F$ defined by $\left\{s_{o}\right\} \times F$. Then the 2-distance function $\delta_{Z}^{2}$ is the constant $s_{o}^{2}$. Hence it is a harmonic function trivially. Consequently, Theorem 3.2 implies that the canonical vector field $Z^{T}$ is always incompressible.

Corollary 4.3. Let $\tilde{M}=I \times{ }_{s} S^{m-1}$ be the warped product of $I=(0, \infty)$ and the unit $(m-1)$ sphere $S^{m-1}$ equipped with the warped product metric $\tilde{g}=d s^{2}+s^{2} g_{S}$. Consider the canonical concurrent vector field $\tilde{Z}=s \frac{\partial}{\partial s}$ on $I \times{ }_{s} S^{m-1}$. Then, for any map $\gamma: I \rightarrow S^{m-1}$, the curve defined by

$$
\psi: I \rightarrow I \times{ }_{s} S^{m-1} ; I \ni s \mapsto(\sqrt{1+2 s}, \gamma(s)) \in I \times{ }_{s} S^{m-1}
$$

has incompressible canonical vector field $Z^{T}$.

Proof. Under the hypothesis, the 2-distance function $\delta_{Z}^{2}$ of the curve $\psi$ given by (4.1) is $\delta_{Z}^{2}=1+2 s$, which is a harmonic function. Consequently, the canonical vector field $Z^{T}$ is incompressible according to Theorem 3.3.

Example 4.1. Consider the map $\gamma: I \rightarrow S^{1}, I=(0, \infty)$, defined by

$$
\gamma(s)=\left(\frac{\cos \sqrt{2 s}+\sqrt{2 s} \sin \sqrt{2 s}}{\sqrt{1+2 s}}, \frac{\sin \sqrt{2 s}-\sqrt{2 s} \cos \sqrt{2 s}}{\sqrt{1+2 s}}\right) .
$$

Then the curve $\psi$ in (4.1) of Corollary 4.3 is given by

$$
\psi(s)=(\sqrt{1+2 s}, \gamma(s)) \in I \times{ }_{s} S^{1} .
$$

Therefore, according to Corollary 4.3, the canonical vector field $\mathbf{x}^{T}=Z^{T}$ is an incompressible vector field.

\section{Acknowledgement}

The author thanks the referee of this article for his/her comments. 


\section{References}

[1] R. Aris, Vectors, Tensors, and the Basic Equations of Fluid Mechanics, Dover Publ., New York, 1989.

[2] R. L. Bishop and B. O’Neill, Manifolds of negative curvature, Trans. Amer. Math. Soc., 145 (1969), 1-49.

[3] B.-Y. Chen, Pseudo-Riemannian Geometry, $\delta$-Invariants and Applications, World Scientific Publishing, Hackensack, NJ, 2011.

[4] B.-Y. Chen, Total Mean Curvature and Submanifolds of Finite Type, 2nd Edition, World Scientific Publishing, Hackensack, NJ, 2015.

[5] B.-Y. Chen, Some results on concircular vector fields and their applications to Ricci solitons, Bull. Korean Math. Soc., 52 (2015), no. 5, 1535-1547.

[6] B.-Y. Chen, Differential geometry of rectifying submanifolds, Int. Electron. J. Geom., 9 (2016), no. 2, 1-8.

[7] B.-Y. Chen, Differential Geometry of Warped Product Manifolds and Submanifolds, World Scientific Publishing, Hackensack, NJ, 2017.

[8] B.-Y. Chen, Addendum to: Differential geometry of rectifying submanifolds, Int. Electron. J. Geom., 10 (2017), no. 1, 81-82.

[9] B.-Y. Chen, Topics in differential geometry associated with position vector fields on Euclidean submanifolds, Arab J. Math. Sci. 23 (2017), no. 1, 1-17.

[10] B.-Y. Chen, Euclidean submanifolds via tangential components of their position vector fields, Mathematics 5 (2017), no. 4, Art. 51, pp. 17.

[11] B.-Y. Chen, Euclidean submanifolds with incompressible canonical vector field, Serdica Math. J., 43 (2017), 321-334.

[12] B.-Y. Chen and S. Deshmukh, Euclidean submanifolds with conformal canonical vector field, Bull. Korean Math. Soc., 55 (2018) (in press).

[13] B.-Y. Chen and L. Verstraelen, A link between torse-forming vector fields and rotational hypersurfaces, Int. J. Geom. Methods Mod. Phys., 14 (2017), no. 12, Art. 1750177, pp. 10.

[14] B.-Y. Chen and S. W. Wei, Differential geometry of concircular submanifolds of Euclidean space, Serdica Math. J., 43 (2017), no. 1, 45-58.

[15] P. A. Davidson, Introduction to Magnetohydrodynamics, 2nd edition, Cambridge University Press, Cambridge, 2017.

[16] J. Marsden and A. Tromba, Vector calculus, 5th Ed., W. H. Freedman and Company, New York, 2003.

[17] M. Matsumoto and K. Eguchi, Finsler spaces admitting a concurrent vector field, Tensor (N.S.), 28 (1974), 239-249.

[18] S.-I. Tachibana, On Finsler spaces which admit a concurrent vector field, Tensor (N.S.), 1 (1950), 1-5.

[19] K. Yano, Sur le parallélisme et la concourance dans l'espace de Riemann, Proc. Imp. Acad. Tokyo, 19 (1943), 189-197.

[20] K. Yano and B.-Y. Chen, On the concurrent vector fields of immersed manifolds, Kodai Math. Sem. Rep., 23 (1971), 343-350.

Department of Mathematics, Michigan State University, East Lansing, MI 48824-1027, USA.

E-mail: chenb@msu.edu 